\section{Intrathyroidal parathyroid adenoma}

Jonette M. Bartlett ${ }^{1}$, Thanh D. Hoang ${ }^{1}$, Alfred F. Shwayhat ${ }^{1}$

1 Department of Internal Medicine, Division of Endocrinology, Naval Medical Center, San Diego.

Intrathyroidal parathyroid adenoma poses a diagnostic challenge when pre-operative localization of the adenoma is desired to guide a minimally invasive parathyroidectomy. Ultrasound-guided fine needle aspiration (UG-FNA) can be used to identify the target tissue. We report a case in which UG-FNA of an intrathyroidal parathyroid adenoma confirmed abnormal parathyroid tissue in a 44 year-old patient who underwent previous bilateral neck exploration which failed to correct her hyperparathyroidism.

\section{Introduction}

Ultrasound- guided fine needle aspiration (UG-FNA) of the parathyroid gland is infrequently performed but can be very useful to distinguish parathyroid adenoma from thyroid tissue. We report a case in which FNA of an intrathyroidal parathyroid adenoma confirmed abnormal parathyroid tissue in a patient who had previously undergone unsuccessful bilateral neck exploration to correct her hyperparathyroidism.

\section{Case Presentation}

A 44 year-old female presented to primary care clinic with a oneyear-history of fatigue, irritability, forgetfulness, and esophageal reflux. She was taking lisinopril for hypertension and atorvastatin for hyperlipidemia. She had no pertinent family medical history. Physical examination was normal. Laboratory results showed serum calcium 11.0 (8.9-10.3 mg/dL), albumin 3.8 (3.5-5.0 mg/dL), PTH intact 167 (ref 10-65 pg/mL), phosphate 2.1 (2.5-4.6 mg/dL), 25-hydroxy-Vitamin D 11 (30-100 ng/mL). Tc-99m sestamibi scan suggested a right inferior parathyroid adenoma (Fig 1A). However, bilateral neck exploration failed to identify the parathyroid adenoma. Postoperative labs showed PTH 184 pg/mL, phosphate 2.2 mg/dL, calcium $11.1 \mathrm{mg} / \mathrm{dL}$. Bedside thyroid ultrasound showed a $9 \mathrm{~mm}$ hypoechoic lesion with increased vascularity (polar vascular pattern) within the inferior portion of the right thyroid lobe (Fig 1B). Repeat sestamibi scan showed a focus of

\author{
Keywords \\ intrathyroidal \\ parathyroid adenoma, \\ hyperparathyroidism, \\ ultrasound-guided fine needle \\ aspiration.
}




\section{Figure $1 \mathrm{~A}$.}

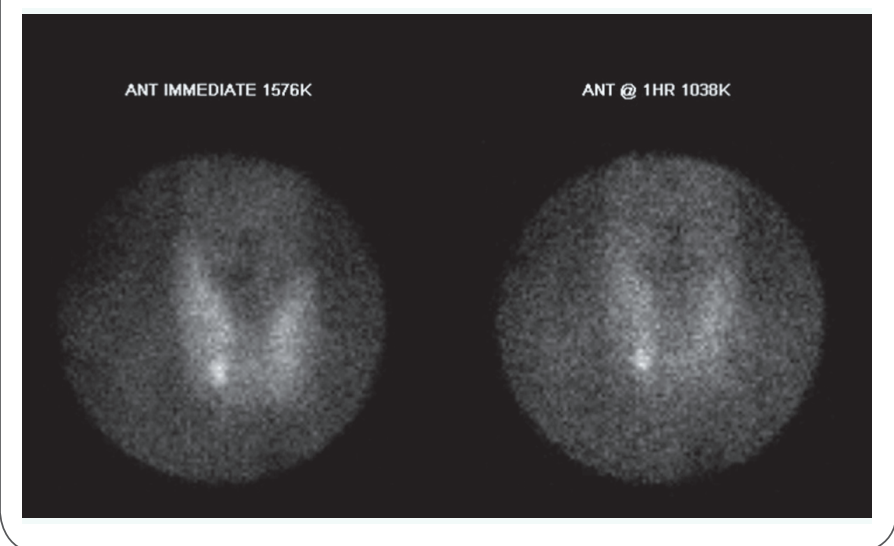

increased accumulation of radiotracer in the inferior pole of the right thyroid lobe. FNA of this lesion showed lymphocytic thyroiditis with cystic degenerative changes (Fig 2A); and PTH measurement from FNA was $4737 \mathrm{pg} / \mathrm{mL}$. The patient then underwent right thyroid lobectomy without complications. Postoperative labs showed PTH 14.9 pg/mL, calcium 9.4. Pathology showed cellular proliferation of clear cells adjacent to normal thyroid follicles containing colloid (Fig 2B). These findings are consistent with intrathyroidal parathyroid adenoma.

\section{Discussion}

Preoperative localization of parathyroid adenoma using ultrasound and sestamibi scan can help to guide a minimally invasive parathyroidectomy, as opposed to a more invasive bilateral neck exploration. When these methods fail to distinguish abnormal parathyroid tissue from thyroid tissue, FNA can be extremely useful. FNA of a parathyroid adenoma can mimic cytological features of lymphocytic thyroiditis; however, aspirates of parathyroid adenoma will show elevated intact parathyroid hormone measurements [1, 2]. It has been reported that cytology and immunocytochemistry may play an important role in the interpretation of US-guided FNA for preoperative localization of parathyroid tissue [3]. If an intrathyroidal parathyroid gland is suspected

\section{Figure 1B.}

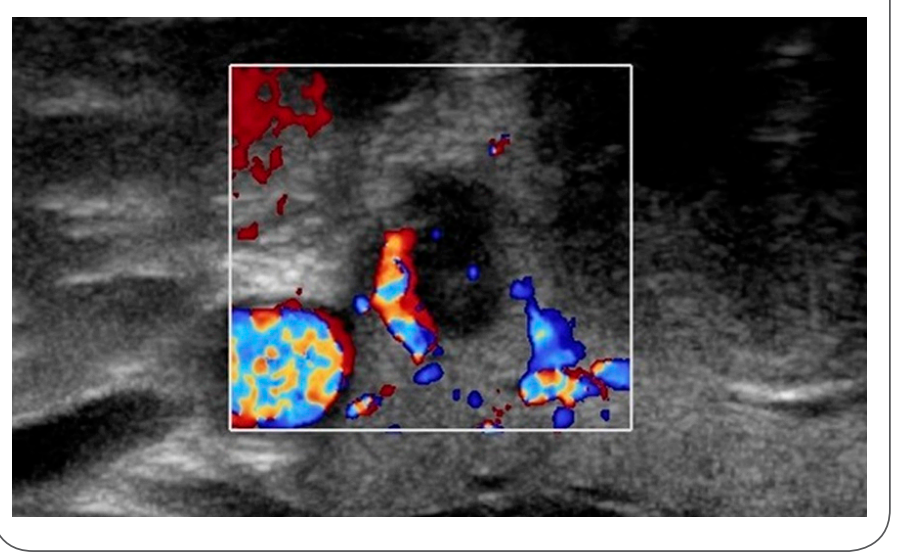

Figure 2A.
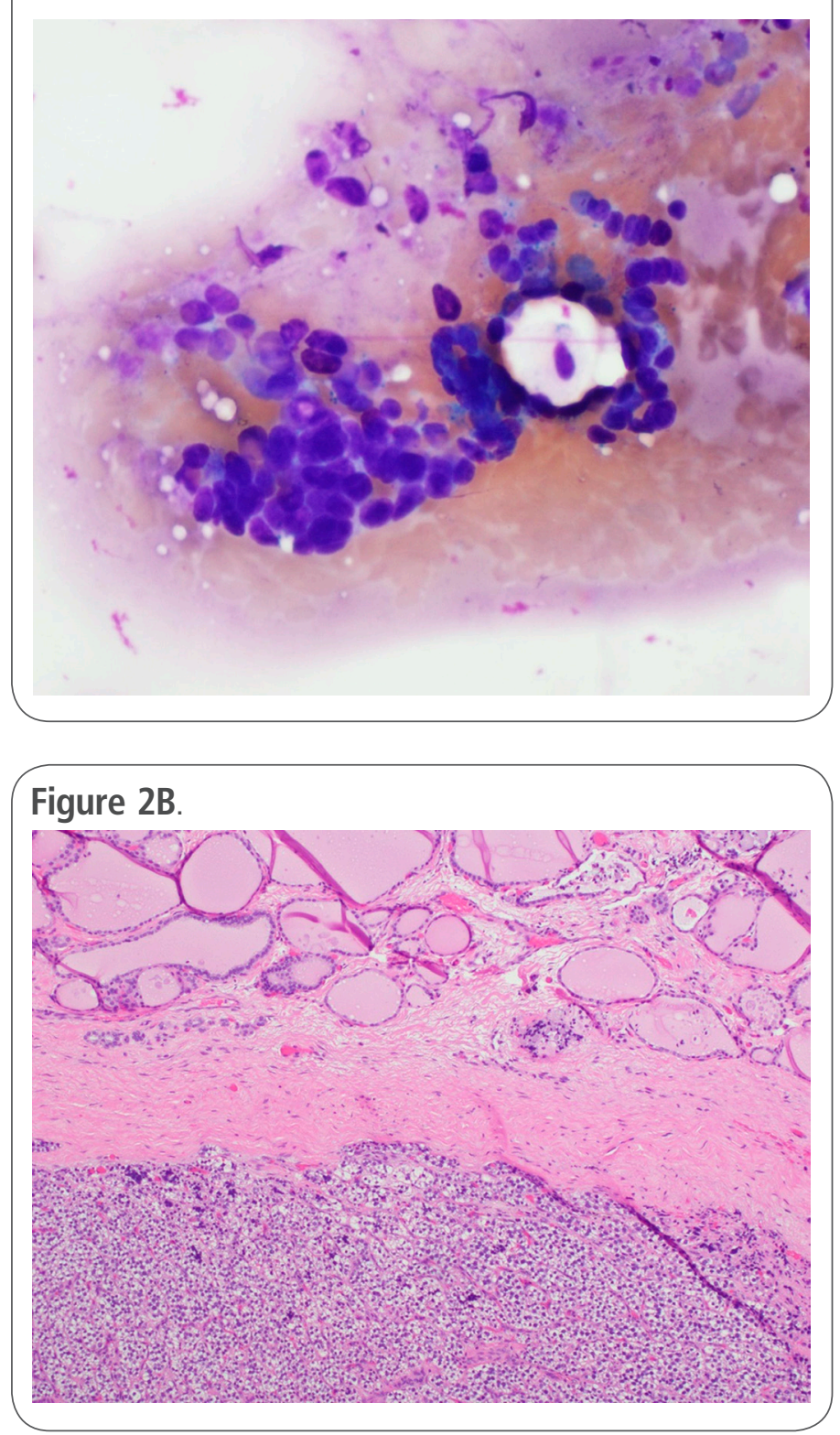
in a patient with primary hyperparathyroidism, an FNA can distinguish adenoma from a thyroid nodule. In this patient, pathology confirmed a $0.6 \mathrm{~cm}$ right inferior intrathyroidal adenoma with otherwise benign thyroid tissue. This case demonstrated the usefulness of FNA of parathyroid lesion in a patient with primary hyperparathyroidism and an intrathyroidal parathyroid adenoma who had failed prior bilateral neck exploration. It may be important to perform a combined approach of cytology and PTH measurement (immunochemistry) in diagnosing and localizing parathyroid tissue.

\section{Disclaimer}

The views expressed in this article are those of the authors and do not necessarily reflect the official policy or position of the Department of the Navy, Department of Defense, or the U.S. Government.

We certify that all individuals who qualify as authors have been listed; that each has participated in the conception and design of this work, the analysis of data, the writing of the document, and the approval of the submission of this version; that the document represents valid work; that if we used information derived from another source, we obtained all necessary approvals to use it and made appropriate acknowledgements in the document; and that each takes public responsibility for it.

\section{References}

1. Auger M, Charbonneau M, Huttner I. Unsuspected intrathyroidal parathyroid adenoma: mimic of lymphocytic thyroiditis in FNA specimens- A Case Report. Diagnostic Cytopathology. 1999; 21: 4: 276-279.

2. Absher KJ, Truong LD, Khurana KK, Ramzy I. Parathyroid cytology: Avoiding diagnostic pitfalls. Head Neck. 2002; 24(2): 157-164.

3. Abati A, Skarulis MC, Shawker T, Solomon D. Ultrasound-guided fine-needle aspiration of parathyroid lesions: a morphological and immunocytochemical approach. Hum Pathol. 1995; 26(3): 338-43.

\section{Comment on this article:}

\section{$4[8$ in $8+2.9$}

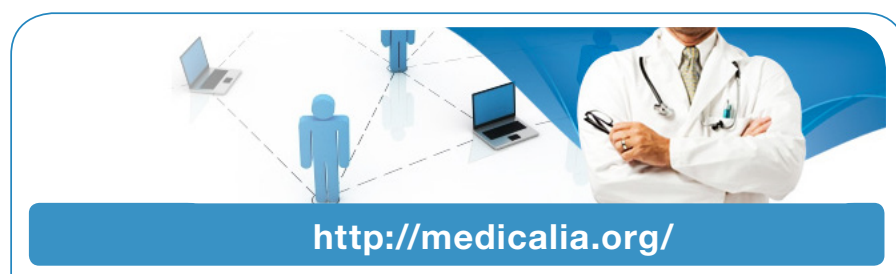

Where Doctors exchange clinical experiences, review their cases and share clinical knowledge. You can also access lots of medical publications for free. Join Now!

\section{Publish with iMedPub}

\section{http://www.imed.pub}

International Archives of Medicine is an open access journal publishing articles encompassing all aspects of medical science and clinical practice. IAM is considered a megajournal with independent sections on all areas of medicine. IAM is a really international journal with authors and board members from all around the world. The journal is widely indexed and classified Q1 in category Medicine. 